\title{
artec Forschungszentrum
}

Nachhaltigkeit

Hellmuth Lange, Andreas Rau

\section{Parken zwischen Ökologie, Ökonomie und Gerechtigkeit.}

Der Streit um kostenpflichtige Firmenparkplätze aus der Sicht von Beschäftigten

artec-paper Nr. 73

November 1999

ISSN 1613-4907

\section{(U) Universität Bremen}

artec | Forschungszentrum Nachhaltigkeit

Enrique-Schmidt-Str. 7

Postfach 330440

28334 Bremen 

Dieser Artikel bildet einen Preprint des gleichnamigen Kapitels der Veröffentlichung:

Hellmuth Lange (Hrsg.)

Ökologisches Handeln als sozialer Konflikt.

Über Grenzen der Moralisierbarkeit der Kluft zwischen Umweltbewußtsein und Umwelthandeln

Opladen (Leske + Budrich) 2000 
Inhaltsverzeichnis

1 Kontexte 3

2 Die Akteure: Wer verfolgte welche Ziele? Wer gab welche Begründungen? 5

3 Phase I: Die Einführung der Parkgebühren $\quad 7$

4 Phase II: Die Bewährung des Reglements im Alltag 9

4.1 Umweltbewußtsein und die Kluft zwischen Leistung und Gegenleistung 10

4.2 Die technische Umsetzung des Projekts 12

4.3 Die argumentative Nachvollziehbarkeit des Projekts 15

5 Ökologische Begründungen als Delegitimierungspotentiale 18

6 Literaturverzeichnis $\quad 21$ 


\section{Parken zwischen Ökologie, Ökonomie und Gerechtigkeit. Der Streit um kostenpflichtigeFirmenparkplätze aus der Sicht von Beschäftigten}

\section{Kontexte}

Nimmt man den erreichten Stand der umweltsoziologischen und umweltpsychologischen Forschung ernst, so ist bei der Analyse umweltbezogener Handlungsprobleme mindestens von zwei Grundsachverhalten auszugehen (etwa de Haan/Kuckartz 1996 und Preisendörfer 1996 für die Soziologie oder Schahn/Giesinger 1993 und Homburg/Matthies 1998 für die Psychologie). Erstens: Umweltprobleme treten als handlungspraktische Herausforderungen von Bürgerinnen und Bürgern in der Regel nie isoliert von anderen Problemen des Alltags auf: des häuslich-außerhäuslichen Alltags der Reproduktion ebensowenig wie des beruflichen Alltags, der Freizeitaktivitäten und sonstiger Felder der Alltagsbewältigung und -gestaltung. Sogenanntes Umwelthandeln steht deshalb von vornherein, unabhängig vom subjektiven Dafürhalten der Individuen, in mehr oder minder komplexen multifokalen Bezugszusammenhängen. Eines der Kernprobleme umweltbezogener Handlungsabsichten besteht in der Folge darin, daß umweltbezogene Handlungsherausforderungen stets in mehr oder minder starker Konkurrenz zu anderen Herausforderungen des Handelns stehen, ohne daß es jedoch möglich wäre, diese Konkurrenz ohne weiteres zugunsten des Umweltaspekts aufzulösen .

Zweitens: In entsprechenden Zusammenhängen bildet das individuelle Handeln von Personen immer nur die eine Seite der Medaille. Die andere Seite besteht darin, daß dieses Handeln in soziale Geflechte von anderen Personen, Gruppen und Institutionen eingebunden ist und von ihnen unterstützt oder gebremst und in jedem Falle beeinflußt wird (Fuhrer 1996; Brand 1997). In diesem durchaus überindividuellen Rahmen entsteht das komplexe Gefüge sozialer Handlungszumutungen, die die Umweltproblematik zu einer Herausforderung des Handelns neben anderen machen und die gleichzeitig dieser Herausforderung ihren je spezifischen Stempel aufdrücken. Die Individuen müssen sie in ihrer jeweils vorfindlichen Form in ihre Alltagsgestaltung mit ihrem Einerseits und Andererseits von persönlichen Wünschen und Abneigungen, von geteilten Überzeugungen und zu respektierenden Normen, von praktischen Möglichkeiten und Grenzen, von Eindeutigkeiten und von Ambivalenzen integrieren (Jurczyk/Rerrich 1993; Poferl/Schilling/Brand 1997).

Umweltaspekte können daher auch subjektiv stets nur einen Relevanzpunkt neben mehreren anderen darstellen, und oft nicht einmal den wichtigsten. Andererseits sollte ihr Gewicht nicht unterschätzt werden. Auch dann, wenn Umweltaspekte in alltagspraktischen Entscheidungen nicht gleichsam in der ersten Reihe der Entscheidungskriterien sitzen, sind sie nicht etwa irrelevant oder abwesend (Preisendörfer 1996; Warsewa 1997), sondern sie laufen bei der Wahrnehmung und Bewertung gesellschaftlicher Handlungszumutungen und Handlungsmöglichkeiten in der zweiten, dritten oder vierten Reihe mit, und in entsprechenden Situationen können sie - in der einen oder anderen Form - auch schnell in die erste Reihe überwechseln. Umweltbewußtsein bleibt insofern eine relevante Bezugs- 
größe individuellen Handelns, auch wenn dies nicht in jeder konkreten Situation zu erkennen ist.

Eine mindestens ebenso wichtige Größe wie das Umweltbewußtsein von Personen stellen deren soziale Angemessenheitsvorstellungen (Bierhoff 1992; Reichle 1998) dar, nicht zuletzt in bezug auf die Bewertung von umweltbezogenen Handlungszumutungen, denen sich die Personen ausgesetzt sehen. Solche Angemessenheitsvorstellungen sind ihrerseits abhängig von kulturellen Traditionen, eigenen Erfahrungen, institutionellen Rahmungen, gruppenspezifischen und situativen Gegebenheiten, verfügbaren materiellen und kulturellen Ressourcen und anderem mehr. Ihren Kern bilden Reziprozitätserwartungen in bezug auf die Leistungen sozialer „Mitspieler“ in der unvermeidlicherweise arbeitsteiligen Bearbeitung konkreter Fragen des eigenen Lebens. Mangelnde umweltbezogene Handlungsbereitschaft von Personen hat ihren Grund vielfach nicht in unzulänglich ausgebildetem Umweltbewußtsein oder in mangelnder Bereitschaft, aus dem sehr wohl vorhandenen Umweltbewußtsein mehr oder minder aufwendige Konsequenzen zu ziehen, sondern in dem Gefühl, daß die eigenen Angemessenheitsvorstellungen im Wechselspiel der beteiligten Akteure verletzt werden.

Der Zusammenhang zwischen beidem - zum einen der Einbettung von Umweltfragen in andere sachliche und normative Bezüge und zum anderen der Relevanz von Angemessenheitsvorstellungen als einem moderierendem Moment der Entwicklung von (unter anderem umweltrelevanten) Handlungsabsichten und dem, was in der Praxis daraus wird - soll im folgenden an einem speziellen Ausschnitt aus der Problematik des Berufsverkehrs dargelegt werden: der Einführung einer sogenannten „Parkplatzbewirtschaftung“ an einer mittelgroßen Universität (ca. 18.000 Studierende, 1.700 wissenschaftliche und 1.100 nichtwissenschaftliche Beschäftigte) in einem norddeutschen Stadtstaat. Während die Parkplätze - es handelt sich um eine Campus-Universität - bis 1997 kostenfrei genutzt werden konnten, wurde ab April 1997 eine Gebühr erhoben. ${ }^{1}$ Umweltgesichtspunkte spielten insofern eine Rolle, als eine nicht unerhebliche Gruppe der NutzerInnen ${ }^{2}$ der Parkplätze aus Berufspendlern besteht. Sie produzieren während der Pkw-Fahrt zur Arbeit in charakteristischer Weise Emissionen und Lärm. Sie belasten damit sowohl die natürliche Umwelt als auch die soziale Umwelt, in letzterem Fall vor allem die Wohn- und Lebensqualität der am städtischen Zielort verbliebenen Städter. Es handelt sich im Falle der Parkplatzbewirtschaftung jedoch nicht um ein reines Umweltproblem. Gerade insofern erscheint es aber typisch für viele Konstellationen, in denen Bürgerinnen und Bürger mit umweltbezogenen Argumenten konfrontiert werden und ihrerseits umweltrelevante Entscheidungen treffen. Es läßt sich zeigen, in welcher Weise vorhandene umweltrelevante Handlungsbereitschaften der Pkw-NutzerInnen ungenutzt bleiben oder gar untergraben werden, sofern deren Angemessenheitsvorstellungen verletzt werden.

Es verblieben zunächst einige öffentliche Straßen, auf denen weiterhin kostenfrei geparkt werden konnte. Ferner stand noch eine Reihe von Rest- und Randflächen zur Verfügung, die zwar nicht zum Parken vorgesehen waren, aber gewohnheitsmäßig für Parkzwecke genutzt wurden.

Hier und im folgenden ist von einem Personenkreis die Rede, der zu fast gleichen Teilen aus Männern und Frauen besteht. Die vorzustellenden Befunde variieren zwischen beiden Teilgruppen kaum. Es geht also um Bewertungs- und Verhaltensmuster von Männern und von Frauen. Um das sichtbar zu machen, benutzen wir an entsprechenden Stellen das sperrige große I. 


\section{Die Akteure: Wer verfolgte welche Ziele? Wer gab welche Begründungen?}

Die Initiierung, Entwicklung und Umsetzung der sogenannten Bewirtschaftung wurde durch verschiedene Akteure mit entsprechend unterschiedlichen Interessen ins Werk gesetzt.

Am Anfang stand ein Beschluß der Landesregierung im Jahre 1995. Er sah vor, daß die Nutzung der Parkflächen öffentlicher Enrichtungen, unter anderem im Bildungsbereich, durch deren Beschäftigte künftig nur noch gegen Gebühr erlaubt sein sollte. Die Maßnahme stand im Rahmen eines umfassenderen Konzepts der Stadtentwicklung, dessen Ziel vor allem darin bestand, Berufspendler dazu zu drängen, stärker auf öffentliche Verkehrsmittel zu setzen, um auf diese Weise die Stadt von privatem Pkw-Verkehr zu entlasten. ${ }^{3}$ Mit der Bewirtschaftung der Parkplätze öffentlicher Einrichtungen sollten die öffentliche Hand respektive die dort Beschäftigten ihren Beitrag zur übergreifenden Zielstellungen leisten. Die Argumentation war also primär verkehrspolitisch; sie war jedoch ihrerseits in eine stadtentwicklungpolitische Argumentation eingebettet. Dahinter standen einerseits ökonomische Erwägungen (Bindung von Kaufkraft an die Stadt. Die Gebühren selbst mögen auch eine Rolle gespielt haben. Wie sich aber zeigen wird, sind sie wegen ihrer geringen Höhe gänzlich ungeeignet, nennenswert zur finanziellen Stabilisierung eines Stadtstaates beizutragen). Insofern die Zurückdrängung von Berufspendlern eine partielle Reduktion des Pkw-Verkehrsaufkommens und eine entsprechende Abschwächung seiner Folgen in Gestalt von Lärm und Emissionen bedeutet, besaß die verkehrspolitische Konzeption andererseits auch eine umweltpolitische Zielstellung.

Die Universität als zweiter Akteur hatte den Beschluß als nachgeordnete Behörde für ihren Bereich umzusetzen. Neben den genannten verkehrspolitischen Gründen, die die mit der Umsetzung betraute Verwaltung übernahm, wurde der Gebühr hier nun eine eigene ökonomische Bedeutung beigemessen. Als Folge seines sinkenden finanziellen Bewegungsspielraums hatte das Land über mehrere Jahre eine einigermaßen rigide Sparpolitik betrieben, die auch die Universität dazu zwang, in beträchtlichem Umfang Kosten einzusparen. In der ihr aufgegebenen Parkplatzbewirtschaftung sah die Universität nun eine Möglichkeit, um sich ihrerseits von ungeliebten Kosten zu befreien: Für die Instandhaltung und Pflege der damals rund 3.000 universitätseigenen Parkplätze auf sechs Stellflächen waren aus dem Universitätshaushalt immerhin rund DM 300.000,- pro Jahr zu erbringen. Die Bewirtschaftung versprach nun eine Möglichkeit, diese Kosten auf die NutzerInnen zu überwälzen und die freiwerdende Summe (mit Billigung des Landes) für Lehr- und Forschungszwecke einzusetzen. Neben dem finanziellen Vorteil, den eine solche Regelung versprach, ließ sie sich zusätzlich als Beitrag zu mehr Gerechtigkeit im Sinne des Verursacherprinzips interpretieren („Wer Kosten verursacht, soll sie auch tragen“!). Mußten doch nun nicht mehr alle Universitätsangehörigen indirekt für etwas aufkommen, das nur von einem Teil von ihnen in Anspruch genommen wurde. Diesem möglichen Vorteil stand jedoch die - wie sich zeigen sollte - nicht unbegründete Befürchtung entgegen, daß eine solche Verlagerung von Kosten von den davon Betroffenen keines-

Allerdings ging es dabei keineswegs um eine generelle Reduzierung des Pkw-Verkehrs, sondern um eine Veränderung der Relation von Berufspendlern einerseits und Einkaufspendlern andererseits. Letztere sollten nicht etwa auch zurückgedrängt, sondern ermutigt werden, ihre Einkäufe weiterhin in der Stadt zu tätigen, um auf diese Art und Weise den Abwanderungsprozeß von Kaufkraft zu bremsen, der die Finanzktaft des Landes in empfindlicher Weise beeinträchtigte (und noch beeinträchtigt). 
wegs mit Begeisterung aufgenommen werden würde. In dieser Konstellation spielte ein dritter Akteur eine wichtige Rolle: der Allgemeine Studentenausschuß (AStA).

In der Hoffnung, daß die Gebühr einen Teil der Beschäftigten und Studierenden davon abhalten würde, weiterhin mit dem Auto zur Universität zu kommen und daß sie anstelle dessen zur Nutzung öffentlicher Verkehrsmittel bewegt werden könnten, unterstützte der AStA die Parkplatzbewirtschaftung als eine ökologisch relevante Maßnahme. Neben stadtentwicklungspolitischen, verkehrspolitischen und (im einzelnen zwischen Landesregierung und Universität durchaus unterschiedlich gerichteten) ökonomischen Erwägungen sowie den von der Universitätsverwaltung ins Spiel gebrachten gerechtigkeitspolitischen Argumenten gehörten somit also auch ökologische Erwägungen zum Fächer der Begründungen. Während der Umweltaspekt in den - insgesamt eher spärlichen - Verlautbarungen der Universitätsverwaltung im Verhältnis zu den ökonomischen Begründungen nur einen Nebenaspekt bildete (immerhin nahm sie ihn aber ausdrücklich auf), bildete er in der Argumentation des AStA das Hauptargument.

Den vierten Akteur in der Runde bildete eine private Parkhausgesellschaft, die mit der technischen Durchführung der Bewirtschaftung betraut wurde. Sie sah sich allerdings von Anfang an in einer Zwickmühle. Als privates Unternehmen war ihr Interesse naturgemäß primär ökonomisch. Insofern war sie mehr als alle anderen Beteiligten an hohen Gebühren interessiert. Um eine möglichst breite Akzeptanz der neuen Regelung zu erreichen, mußte sie hingegen eher niedrige Gebühren ins Auge fassen. Diese Situation konnte die Universität dahingehend nutzen, daß sie die endgültigen Gebühren auf weniger als ein Drittel dessen herunterhandelte, was von der Parkhausgesellschaft zunächst gefordert worden war. Aus der Sicht der prospektiven Kunden war dies ein erfreuliches Ergebnis. Dem stand jedoch als Kehrseite des gelungenen Handels ein Nachteil in technischer Hinsicht entgegen. Die gesamte Installation - vor allem die Schrankenanlage ${ }^{4}$ und die Automaten, in die das Geld für die Parkscheine eingeworfen werden muß, und aus denen sodann die Parkscheine entnommen werden können - mußte möglichst billig gehalten werden, damit sich das ganze Unterfangen für die Parkhausgesellschaft überhaupt noch rechnete. Infolgedessen entschied sich die Parkhausgesellschaft für die Aufstellung von Automaten mit einem relativ begrenzten Funktionalitätsspektrum. Außerdem weisen die Automaten ergonomische Mängel auf.

Als fünfter Akteur im Bunde ist die Firma zu nennen, die die Automaten herstellt, aufstellt und wartet. Diese Firma wäre aus ökonomischem Interesse und angesichts ihres technischen Könnens gerne bereit gewesen, teurere und kompliziertere Automaten zu liefern. Sie begnügte sich dann jedoch notgedrungen mit dem Spatz in der Hand, weil die Taube auf dem Dach unter den gegebenen Randbedingungen nicht zu haben war.

Die Akteurkonstellation war also komplex, und das Spektrum der genannten Ziele der Maßnahme war breit. Umweltpolitische Ziele spielten teils direkt (Universitätsverwaltung, AStA) und teils indirekt (Landesregierung) eine Rolle, mit Ausnahme des AStA jedoch nur als ein Gesichtspunkt neben anderen und letztlich entscheidungsrelevanteren Gründen.

Die Parkplätze sind durch Schranken versperrt. Vor den Schranken steht ein Automat, an dem Parkscheine gelöst werden. Ist dieser Vorgang abgeschlossen, öffnet sich die Schranke für den kurzen Zeitraum der Durchfahrt eines Autos. Für die nachfolgenden Autos wiederholt sich der Vorgang. Die Ausfahrt ist frei. 
Man einigte sich schließlich auf eine Gebühr von DM 1,10 pro Tag für das Parken auf unbedachten Parkflächen und DM 2,20 auf überdachten Parkflächen. Diese Beträge sind im Verhältnis zu dem, was teilweise an anderen Universitäten gefordert wird (CampusUniversitäten eingeschlossen), niedrig (HUI 1996). Das gilt um so mehr im Vergleich mit den Parkkosten auf den Flächen anderer Betriebe oder gar auf öffentlichen Parkflächen in Innenstadtbereichen.

In einer ersten Abschätzung konnte daher durchaus angenommen werden, daß die Maßnahme akzeptiert werden würde: Das soziokulturelle Milieu einer Universität ließ eine eher überdurchschnittliche Aufgeschlossenheit für die ökologisch relevanten Aspekte der Maßnahme erwarten. Der vergleichsweise niedrige Betrag der Gebühren versprach, den gleichwohl zu gewärtigenden Ärger über die Tatsache, daß überhaupt eine Gebühr eingeführt wurde, in engen Grenzen zu halten. Der Zusatzeffekt einer Kostenentlastung der Universität und der dadurch ermöglichte Zuwachs der Mittel für Lehre und Forschung als den Kernaufgaben einer Universität versprach weitere eskalationshemmende Wirkungen. Parkhausgesellschaft und Universitätsverwaltung als die Hauptverantwortlichen für den unmittelbaren Einführungsprozeß gaben sich denn auch überzeugt, daß nach anfänglichem Murren ein Gewöhnungsprozeß einsetzen würde, der zur Akzeptanz der Maßnahme führen werde - hatte man aus ihrer Sicht doch alles getan, um den neuen Kunden die Sache möglichst leicht zu machen.

\section{Phase I: Die Einführung der Parkgebühren}

Um die Reaktionen der Beschäftigten und Studierenden kennenzulernen, wurde 14 Tage nach Einführung der Maßnahme eine Befragung durchgeführt (Befragung 1997). ${ }^{5}$ Diese Befragung fand (im Unterschied zu einer weiteren Befragung nach Ablauf von zwei Jahren) noch ohne die Unterstützung der Universitätsverwaltung und der Parkhausgesellschaft statt. Obwohl einerseits beide Seiten davon überzeugt waren, alles versucht zu haben, um den Kunden den Einstieg so leicht wie möglich zu machen, bestand andererseits - ebenfalls auf beiden Seiten - eine diffuse Sorge, daß die Kunden das schließlich erreichte Angebot vielleicht doch nicht angemessen würdigen könnten. Derlei Befürchtungen sind schwer zu fassen. Soweit sich Beteiligte gesprächsweise dazu äußerten, wurden allgemeine Vermutungen und Überzeugungen des folgenden Typus genannt: „Die melden sich immer nur, wenn etwas mal nicht klappt. Den Rest nehmen sie mit, als wenn das alles selbstverständlich wäre“; „Denen kann man doch sowieso nichts recht machen ";. und nicht zuletzt: "Die reden doch alle nur von Umwelt, und wenn es dann praktisch wird, dann kneifen sie ". ${ }^{6}$ Vor dem Hintergrund solcher Überzeugungen bestand die Sorge, daß eine Befragung über möglichen Unmut - gewollt oder ungewollt - selbst zur Beförderung von Unmut beitragen würde, der den eigentlich zu erwartenden Gewöhnungsprozeß unnötig in die Länge ziehen oder gar unterminieren könnte.

\footnotetext{
5 Der Rücklauf betrug 29\% (N=302). Es wurden Personen unterschieden, die „Immer“, „Oft“, „Manchmal“ oder „Nie“ mit dem PKW, mit Bahn und Bus und mit dem Rad zur Universität kamen. 75 \% der Befragten verfügten über ein Auto. - An der Vorbereitung dieser Befragung war Esther Bernds beteiligt.

6 Zumindest die letzte der genannten Äußerungen liest sich wie eine populäre Fassung der in der Wissenschaft so anhaltend behandelten „Kluft zwischen Umweltbewußtsein und Umweltverhalten“.
} 
Wie fielen die Reaktionen der zu Kunden gewordenen Beschäftigten und Studierenden tatsächlich aus? Die Ablehnung hätte kaum größer sein können. Die Maßnahme wurde von

$82 \%$ derjenigen negativ eingestuft, die „Immer“ mit dem Auto zur Universität kommen. ${ }^{7}$ Zwar kündigten immerhin $17 \%$ an, künftig ihre Verkehrsmittelnutzung zu überdenken. ${ }^{8}$ Gleichzeitig kündigten aber 66 \% der Immer-FahrerInnen an, daß sie versuchen würden, die Bezahlung der Gebühren zu umgehen. ${ }^{9}$

Damit scheint sich zu bestätigen, daß immer dann, wenn es ans Geld geht, die Handlungsbereitschaft von Akteuren rapide abnimmt, und zwar auch dann, wenn - wie im vorliegenden Fall - ökologische Gründe ein zentrales Element (neben anderen) der Begründung darstellen und wenn die betreffenden Personen ihrerseits ökologische Gründe als ausgesprochen wichtig einschätzen. Scheinbar besteht dieser Effekt sogar dort, wo der tatsächlich zu erbringende Betrag absolut und im Vergleich zu anderen Gelegenheiten, bei denen Parkgebühren zu entrichten sind, ausgesprochen gering ausfällt. „Low Cost"! als kategorischer Imperativ?“

Bei näherem Hinsehen erweist sich diese Deutungsmöglichkeit als weniger plausibel, als es zunächst scheinen mag. Immerhin erklärten selbst $40 \%$ derer, die eine Gebühr zwischen

DM 1,- und DM 1,50 als für sie akzeptabel benannten (mithin einen Betrag exakt in jenem Bereich, in dem die Gebühr zu erbringen ist) ${ }^{10}$ daß sie versuchen würden, die Parkplatzregelung zu umgehen. Für die hier deutlich werdende Möglichkeit, daß es bei der Ablehnung nicht immer beziehungsweise nicht in der Hauptsache ums Geld gehen muß, spricht ein weiteres Ergebnis: Die Immer-FahrerInnen bewerteten die verlangten Gebühren zu $61 \%$ mit „o.k.“, weitere $5 \%$ bewerteten sie als „Niedrig“. ${ }^{11}$ Zwei Drittel räumen also ein, daß sich die Höhe der Gebühren gewissermaßen in zivilisierten Bahnen hält. Wieso kommt es trotzdem zu einer so breiten Ablehnung der Maßnahme?

Eine überraschende erste Erklärung läßt sich aus folgendem Befund gewinnen: Von denjenigen Befragten, die über ein Auto verfügten, ${ }^{12}$ und ein monatliches Einkommen von weniger als DM 4.500,- bezogen, hatten fast zwei Drittel (62 \%) bis zum Zeitpunkt der Einführung der Parkgebühren überhaupt nie mehr als DM 10,- monatlich für Parkgebühren gezahlt. Weitere $20 \%$ von ihnen hatten bis dahin monatlich nicht mehr als DM 20,für Parkgebühren aufzuwenden. Diese geringe Höhe der Belastungen durch Parkgelder vor Einführung der Gebühr fand sich sowohl in der unteren Einkommensgruppe (bis DM 2.000,-) wie auch in der mittleren (DM 2.000,- bis DM 4.500,-) der insgesamt drei unterschiedenen Gruppen. Insgesamt hatten also $80 \%$ der Befragten bis zur Einführung der Bewirtschaftung nicht mehr als DM 20,- monatlich an Parkgebühren zu zahlen. Auf in

7 Der Bewertung der folgenden Befunde lag, sofern nichts anderes angegeben ist, eine Viererskala zugrunde, in der zwischen „Sehr schlecht", „Schlecht“, „Gut" und "Sehr gut“" gewählt werden konnte. „Negativ“, wie oben im Text, bezeichnet die Summe der Antworten zu den beiden „Schlecht"-Varianten. Die Ablehnungen waren hier und bei den meisten folgenden Items in der Gruppe der Oft-FahrerInnen noch größer als bei den Immer-FahrerInnen. Wir beschränken uns im folgenden aber aus Gründen der Übersichtlichkeit und der Vergleichbarkeit der Zahlen auf die Gruppe derer, die „Immer“ mit dem Auto zur Universität kommen.

8 In der Gruppe der Oft-FahrerInnen waren es sogar $43 \%$.

9 Das war zum damaligen Zeitpunkt vor allem dadurch möglich, daß noch längst nicht alle Stellen, an denen Autos geparkt werden konnten, entweder versperrt oder eben kostenpflichtig gemacht waren (siehe Fußnote 1).

auf unbedachten Parkplätzen.

Die dritte mögliche Bewertung lautete „Hoch“. Sie wurde nur von $33 \%$ gewählt.

In diesem Fall ist die Trennung in Immer-FahrerInnen, Oft-FahrerInnen und Manchmal-FahrerInnen aufgegeben. 
etwa diesen Betrag beläuft sich aber die neue Gebühr, wenn man einen fast täglichen Besuch der Universität zugrunde legt.

Dieses Ergebnis mag erklären, weshalb in den betreffenden beiden Einkommensgruppen zwei Drittel der Befragten erklärten, „die paar Mark“ fielen für sie finanziell „sehr wohl ins Gewicht", obwohl sich die Gebühren in absoluten Beträgen und im Vergleich zu anderen regelmäßigen Ausgaben wie Kino und Gaststättenbesuche (nach denen ebenfalls gefragt wurde) doch sehr im Rahmen halten: Es ist möglicherweise gar nicht der absolute Betrag, sondern der relative Unterschied zwischen den Gegebenheiten vor der Einführung der Parkgebühren und den Gegebenheiten nach deren Einführung. Der Unterschied beträgt gewissermaßen $100 \%$.

Wenn tatsächlich weniger die Höhe der Gebühren als die Ungewohntheit der neuen Situation zu den negativen Voten geführt hätte, dann wäre das auch als eine Bestätigung der beiden Hauptverantwortlichen für die konkrete Gestaltung des Wandels zu lesen, die von vornherein auf eine bald einsetzende Gewöhnung gesetzt hatten: Neue Situationen sind als solche naturgemäß nicht von Dauer. Die Vermutung, daß sich der anfängliche Ärger im Laufe der Monate auflösen würde, schien sich denn auch relativ schnell zu bestätigen: Die vormals gut angenommenen Parkplätze wurden unmittelbar nach der Einführung der Gebühren tatsächlich in erheblichem Umfang gemieden; nach zwei, drei Monaten füllten sie sich jedoch wieder, und die Nutzung erreichte schließlich wieder in etwa das vorherige Niveau. Allerdings waren in der betreffenden Zeit auch zunächst noch verbliebene nicht gebührenpflichtige Parkmöglichkeiten durch die Aufstellung von Verbotsschildern und verhältnismäßig rigide Kontrollen als Ausweichmöglichkeiten abgeschnitten worden. Insgesamt schien sich damit aber schließlich doch zu bestätigen, was die lokale Tageszeitung schon unmittelbar nach der Einführung meinte herausgefunden zu haben: „So richtig verärgert war eigentlich niemand“" (XYZ-Kurier vom 22.04.1997).

Andererseits wurden immer wieder zum Teil massive Klagen von NutzerInnen der Parkplätze laut. Sie bezogen sich auf eine ganze Palette von Einzelpunkten des Parkreglements. Angesichts dieser Situation entschlossen sich die Parkhausgesellschaft, der Automatenlieferant und die Universitätsverwaltung zwei Jahre nach der Einführung der Kostenpflichtigkeit, nun selbst eine Befragung in Auftrag zu geben, die die wichtigsten Probleme, die Häufigkeit ihres Auftretens und das erreichte Akzeptanzniveau des Reglements ermitteln sollte (Befragung 1999). ${ }^{13}$

\section{Phase II: Die Bewährung des Reglements im Alltag}

Zwei Jahre nach der Einführung der Parkplatzbewirtschaftung gaben noch immer erst $55 \%$ der Befragten an, sie hätten sich an die Gebühren gewöhnt, und lediglich $25 \%$ hielten die Parkplatzgebühren auf dem Gelände der Universität für gerechtfertigt. Die übrigen $75 \%$ antworteten mit „Nein“. Auch in der Gruppe derer, die angaben, sie hätten sich an die Gebührenregelung gewöhnt, hielten fast zwei Drittel (61 \%) die Erhebung von Gebühren für nicht gerechtfertigt. Von Akzeptanz kann also keine Rede sein; bestenfalls

13 Der Rücklauf betrug $37 \%$ ( $\mathrm{N}=386$ ). Im Unterschied zur Befragung 1997 wurden dieses Mal ausschließlich Personen befragt, die mit dem Auto zur Universität kommen. $80 \%$ der Befragten kommen an vier und mehr Tagen zur Universität. $89 \%$ nutzen dafür das Auto „Immer“ oder „Oft“. 
von resignativer Duldung eines Sachverhaltes, den zu ändern man sich außerstande sieht. Was äußerlich als Gewöhnung erscheint (vor allem die breite Nutzung der Parkplätze trotz ihrer Gebührenpflichtigkeit), erweist sich bei näherem Hinsehen als eine nicht besonders reißfeste Decke über anhaltender Ablehnung. Wo liegen die Gründe?

\subsection{Umweltbewußtsein und die Kluft zwischen Leistung und Gegenleistung}

Schon in der ersten Befragung (1997) hatte es eine Reihe von Hinweisen gegeben, die auf tiefer liegende Ablehnungsgründe als die zunächst ungewohnte Situation und gegebenenfalls die Höhe der Beiträge verwiesen: Dem Statement „Was mich stört, sind nicht die paar Mark, sondern die ganze Art und Weise, in der die Sache angegangen wurde“, stimmten seinerzeit $47 \%$ der Immer-FahrerInnen zu. Und selbst jene, die damals regelmäßig mit Bahn und Bus zum Arbeitsplatz kamen, stimmten dem zitierten Satz in gleicher Breite zu (47 \%) wie die Immer-AutofahrerInnen. Die darin zum Ausdruck kommende Kritik wird - wie im folgenden dargelegt wird - in der Befragung des Jahres 1999 klarer erkennbar. Sie verweist alles in allem auf eine tiefgehende Kluft zwischen dem, was die Parkenden einerseits als angemessene Gegenleistung des Parkplatzbetreibers für ihre eigene Bereitschaft erwarten, sich auf die neuen Bedingungen und Prozeduren der Park platznutzung einzulassen, und dem, was ihnen andererseits im Alltag der Parkplatznutzung tatsächlich geboten wird. Auf diesem Fundament baut die Bewertung der Gebührenpflichtigkeit und des Parkreglements als solchem auf, und auf dieser Grundlage entscheidet sich, in welchem Maße die Parkenden bereit sind, die aus dem Parkreglement erwachsenden Verhaltenszumutungen als ein Element ihres persönlichen Alltags zu akzeptieren. ${ }^{14}$

Bei letzterem spielen nun nicht allein die gegebenen äußeren Zwänge eine Rolle, sondern auch die eigenen Einstellungen und Werthaltungen. Dazu gehören auch jene, die als Elemente von Umweltbewußtsein verstanden werden können und die in dieser Funktion die verkehrspolitischen, stadtentwicklungspolitischen und nicht zuletzt die umweltpolitischen Begründungen betreffen, die die Einführung der Parkplatzbewirtschaftung begleitet und gerechtfertigt haben: $90 \%$ der befragten Immer-FahrerInnen pflichteten schon 1997 dem Statement bei: „Für mich sind ökologische Fragen wichtig“ und $70 \%$ dieser Teilgruppe unterschrieben den Satz „Ich fahre deshalb auch nur mit dem Auto, wenn es anders kaum geht".

Beide Antworten müssen natürlich auch als Antworten im Sinne sozialer Erwünschtheit verstanden werden. Es zeigte sich jedoch, daß die zurückzulegenden Entfernungen beziehungsweise die zur Verfügung stehenden öffentlichen Verkehrsmittel für zwei Drittel dieser Personen tatsächlich kaum eine zumutbare andere Möglichkeit boten, als mit dem Auto zur Arbeit zu kommen. ${ }^{15}$ Insofern erscheint es auch keineswegs inkonsequent, wenn

Es handelt sich hierbei um einen Rückkopplungsprozeß , den Fuhrer/Wölfing als „mentales Coping“ beschreiben (Fuhrer/ Wölfing1997: $71 \mathrm{ff}$.)

15 Nur ein Drittel benötigte weniger als 15 Minuten mit dem Auto. Auch hier kann allerdings nur in einem Teil der Fälle davon ausgegangen werden, daß angemessene ÖPNV-Alternativen zur Verfügung stehen. Das Maß unserer Bewertung bildeten einerseits die Angaben der Befragten über den unterschiedlichen Zeitbedarf für die Bewältigung des Weges zur Arbeit mit dem Auto einerseits und öffentlichen Verkehrsmitteln andererseits. Auch bei großzügiger Berücksichtigung der Erfahrung, daß AutofahrerInnen den Zeitbedarf der von ihnen üblicherweise nicht in Anspruch genommenen öffentlichen Verkehrsmittel überschätzen (Gorr 1996), verblieben für den Fall der Nutzung öffentlicher Verkehrsmittel (soweit überhaupt noch vorhanden) zeitliche Mehrbelastungen von überwiegend mehr als 50\%. Im übrigen haben wir die auf dem Weg zur Universität zurückzulegenden Entfernungen und die auf den betreffenden Strecken zur Verfügung stehenden Alternativen in der Wahl des Verkehrsmittels anhand der Postleitzahlen der Wohnorte der Befragten überprüft. Die Abschätzungen der Befragten erwiesen sich dabei als recht belastbar. 
einerseits ökologische Fragen von fast allen Immer-FahrerInnen als für sie ,sehr wichtig“ erklärt wurden (allgemein), und wenn andererseits nur $9 \%$ angaben, ökologische Gründe seien in ihrer (konkreten) Verkehrsmittelwahl für den Weg zur Arbeit „sehr wichtig““. ${ }^{16}$ Das mit Abstand am häufigsten als ,sehr wichtig“ eingestufte Kriterium bilden hingegen Zeitgründe (94\%). ${ }^{17}$ Der Weg zur Arbeit nimmt eben gerade bei den Immer-FahrerInnen einen erheblichen Teil ihres gesamten Tages in Anspruch: Sie haben die längsten Anfahrtszeiten. Zeitgesichtspunkte im Sinne möglicher Optimierungen des Zeitaufwandes fallen dabei vergleichsweise stark ins Gewicht. Angesichts dessen rücken ökologische Kriterien, soweit sie mit dem Zeitkriterium konkurrieren, auch dann in den Hintergrund, wenn sie als solche durchaus für ,,sehr wichtig“" gehalten werden. ${ }^{18}$

Um herauszufinden, ob diese Wohnort-Arbeitsort-Problematik von den befragten Personen als eine Art Joker genutzt wird, um ökologische Kriterien auf der ganzen Breite ihrer automobilen Alltagssituation zu delegitimieren und in bezug auf mögliche Anforderungen an sie selbst zu neutralisieren, hatten wir in der gleichen Untersuchung (1997) um eine Stellungnahme zum Verursacherprinzip als Begründungsrahmen einer verkehrspolitischen Strategie gebeten, die die AutofahrerInnen mit entstehenden Kosten des Autoverkehrs belastet. (Wegekosten, Umweltbelastungen, Kapazitätsengpässe auf den Straßen). ${ }^{19}$ Eine solche politische Option bewerteten immerhin noch fast die Hälfte der befragten ImmerFahrerInnen positiv, nämlich als ,völlig richtig“ (19 \%) oder als ,da ist was dran“ $(30 \%) ;{ }^{20}$ nur $17 \%$ votierten für die Variante ,völliger Quatsch“. Welche konkreten Bereitschaften hinter derartigen Bekundungen stehen, ist naturgemäß schwer zu sagen, am wenigsten im Rahmen einer isolierten Meinungsbefragung. Daß das Votum der befragten Immer-FahrerInnen nicht als bare Rhetorik anschlußlos im Raume schwebte, läßt sich unter anderem aus dem folgenden Befund zur Bewertung der damals noch neuen Parkgebühr erschließen: Selbst in der Gruppe derer, die weniger als DM 2.000,- im Monat zur Verfügung hatten, wurde die Höhe der Gebühr von $54 \%$ als „o.k. “ bewertet; ${ }^{21}$ in der mittleren Einkommensgruppe (DM 2.000,- bis 4.500,- pro Monat) waren es $68 \%$, in der oberen Gruppe mit Gehältern über DM 4.500,- sogar $75 \%$. Weitere 10\% der Angehörigen dieser dritten Gruppe beurteilten die Gebühr als „niedrig“. Die Zahlen zeigen, daß die tatsächlich recht geringe Höhe der geforderten Gebühren von den NutzerInnen durchaus gewürdigt wurde.

Andererseits ist dieser Befund nicht allzu überraschend: Immerhin bildet der Weg zur Arbeit (oder zur Vorlesung) den am häufigsten getesteten Weg der Befragten - auch für den unvermeidlichen und deshalb durchaus auch geläufigen Fall, daß das üblicherweise genutzte Auto einmal nicht zur Verfügung steht.

Immerhin votierten weitere $21 \%$ für die Antwortvariante ,wichtig“.

17 Die Variante ,wichtig“" wurde von weiteren $4 \%$ gewählt.

18 Demgegenüber wohnen Oft-FahrerInnen durchschnittlich weniger weit entfernt, und sie haben durchschnittlich mehr Möglichkeiten, den Weg mit anderen Verkehrsmitteln zu bewältigen. Das mag auch erklären, weshalb in der Befragung des Jahres 1997 aus dieser Gruppe sogar 43\% angaben, sie würden künftig ihre Verkehrsmittelwahl änders treffen.

19 Wir baten um eine Stellungnahme zu folgender Pressemeldung: „Bundesminister Wissmann forderte unlängst: ,Billiger darf das Autofahren in Deutschland auf keinen Fall werden. Denn wir wollen Verursacherprinzipien auch im Verkehr mehr zur Geltung bringen, und das bedeutet: Anlastung der Wegekosten, Berücksichtigung der Umweltbelastungen und der Knappheit der Kapazitäten auf unseren Straßen“".

Die bald nach diesem Statement aufgebrochene politische Auseinandersetzung um Benzinpreiserhöhungen als Element einer ÖkoSteuer zeigt im übrigen, daß derartige Forderungen nicht im Verhältnis 1:1 an ökologischem Maulheldentum und handlungspraktischem Konservativismus der BürgerInnen in die Krise geraten, sondern zunächst einmal an derartigen Verhaltensmustern der Verantwortlichen des institutionellen politischen Systems: Anstatt die BürgerInnen von der sachlich kaum bestritteten Notwendigkeit der Verteuerung von Energie zu überzeugen und sie für eine entsprechende Politik zu gewinnen zu suchen, haben sich seinerzeit Politiker aller Lager bekanntlich darin zu übertreffen versucht, den BürgerInnen die fragliche Perspektive als Ausbund mangelnden Sachverstands und politisch-praktischer Maßlosigkeit auszureden.

20 Weitere $34 \%$ hielten das Statement lediglich für ,etwas überzogen“.

21 Die möglichen Alternativen lauteten „Hoch“ und „Niedrig“. 
Wenn die Parkplatzbewirtschaftung trotzdem von Anfang an massiv kritisiert wurde und wenn sich daran auch nach zwei Jahren „Gewöhnungszeit“" nicht viel geändert hat, so vor allem deshalb, weil die Leistungen der Parkhausgesellschaft angesichts der Zumutungen, die die Parkplatzbewirtschaftung für die Beschäftigten und Studierenden bedeutet, als unzulänglich angesehen werden. Die finanzielle Zumutung der Gebühren bildet auch 1999 nicht den Hauptpunkt des Unmutes. Die finanzielle Seite der Sache erhält ihre Brisanz erst insofern, wie die zu zahlende Gebühr als eigene Leistung gesehen wird, der keine angemessene Gegenleistung der Betreiberseite gegenübersteht. Welche Leistungen dabei im einzelnen als unzureichend angesehen werden, ist im Laufe der beiden vorangegangenen Jahre immer deutlicher geworden. Die bis heute anhaltende Ablehnung der NutzerInnen rührt in diesem Sinne nicht daher, daß sie noch immer nicht genügend Zeit gehabt hätten, sich an die zunächst neue Regelung zu gewöhnen, sondern daher, daß sie in dieser Zeit Erfahrungen gesammelt haben, die sie überhaupt erst zu der heutigen Form der Akzeptanzverweigerung geführt haben.

Umweltbezogene Handlungsbereitschaften - ohnehin schon abgeglichen mit sonstigen Erfordernissen der Erledigung des eigenen Alltags (in diesem Fall der Bewältigung des Weges zur Arbeit) und in einer Situation, in der Umweltgesichtspunkte für alle Beteiligten bestenfalls einen Teilaspekt der in Frage stehenden Problematik neben anderen darstellen drohen dabei vollends auf der Strecke zu bleiben. Die fraglichen negativen Erfahrungen lassen sich überwiegend als Folgen technischer Probleme beschreiben.

\subsection{Die technische Umsetzung des Projekts}

Die negativen Erfahrungen, die hier gesammelt wurden, lassen sich imn folgendem Satz eines der Befragten zusammenfassen: „,Die Parkgebühren finde ich in Ordnung, aber die technische Realisierung der Parkplatzbewirtschaftung läßt sehr zu wünschen übrig “. Im einzelnen geht es um drei Problemschwerpunkte: erstens um die zu enge Gestaltung der Zuwegung, zweitens um Ausfälle der Automaten, an denen die Parkscheine gelöst werden, und drittens um die Konstruktion und die Programmierung des Geldeinwurfs:

Die Automaten nehmen Münzen jeder Höhe an, sie wechseln aber nicht. Darauf wird zwar mittels eines Schildes hingewiesen. Vor allem Personen, die das Reglement noch nicht kennen, werden aber oft erst darauf aufmerksam, wenn das Geld bereits eingeworfen ist. In diesen Fällen wird für das Parkticket im Wert von DM 1,10 fallweise ein Betrag von DM 2,- oder gar DM 5,- gezahlt. Um das zu vermeiden, empfiehlt es sich, die Beträge von DM 1,10 beziehungsweise DM 2,20 schon abgezählt mitzubringen. Da das aber vor allem im Kreise der Manchmal-FahrerInnen nicht alle wissen oder da selbst diejenigen, die immer mit dem Auto kommen, vor ihrer Abfahrt zur Universität an alles mögliche, nicht aber an das Kleingeld für die Parkuhren denken, suchen sie das nötige Kleingeld zum Teil erst zusammen, wenn sie mit ihrem Auto bereits vor dem Automaten stehen. Auf diese Weise kommt es zu ersten kleinen Stauungen.

Es kommt hinzu, daß der Schlitz für den Geldeinwurf ergonomisch relativ ungünstig plaziert ist. Die Automaten sind ursprünglich für Fußgänger und nicht für sitzende AutofahrerInnen ausgelegt worden. Besser angepaßte und leistungsfähigere Automaten mochte die Betreibergesellschaft angesichts der niedrigen Gebühren aus Sorge um Kostendeckung 
nicht aufstellen. Daher passiert es immer wieder, daß Münzen von den NutzerInnen nicht sogleich in den Geldschlitz praktiziert werden können. Nicht selten fallen sie in der Folge auf den Boden. Da nun aber die Fahrzeuge relativ nahe an die Automaten heranmanövriert werden müssen, damit diese auch von Personen mit kürzeren Armen oder ungünstiger Sitzposition aus dem Auto heraus ,gefüttert“ werden können, ist es kaum möglich, die Autotür zu öffnen, um das herabgefallene Geld wieder aufzuheben. Daher suchen die Autofahrer in solchen Fällen zunächst in ihren Taschen nach weiteren passenden Münzen. Auch das bewirkt Zeitverzug, und die Tendenz zur Staubildung verstärkt sich. Hat jemand jedoch nur die abgezählten Münzen dabei, wird es unerläßlich, die herabgefallene(n) Münze(n) wiederzufinden und aufzuheben oder beim nachfolgenden Parker um finanzielle Nothilfe zu bitten. Derlei ist nicht nur lästig. Es geht auch weitere Zeit verloren, und die schon bestehenden Ansätze zur Staubildung verstärken sich.

Der gleiche Effekt tritt in noch stärkerem Maße auf, wenn Münzen von den Automaten nicht angenommen werden oder wenn die Automaten die Parkberechtigungsscheine in Form kleiner Abschnitte aus Papier nicht ausdrucken. In diesen Fällen öffnen sich folgerichtig die Schranken nicht. Der Extremfall tritt ein, wenn der Automat oder die Schranke gänzlich ausfallen. Für diese Fälle ist zwar eine Signallampe installiert. Sie ist jedoch relativ klein. Zudem fällt auch sie manchmal aus. In allen solchen Situationen wird es erforderlich, den Parkzuweg zu verlassen, um eine andere Parkmöglichkeit zu suchen. Wegen der Enge der Zuwegung ist es aber nur äußerst schwer möglich, individuell aus der Reihe auszuscheren. Noch schwieriger ist es, die Schlange als Ganze zum Rückwärtsfahren zu bewegen. Gleichwohl wird beides im Fall der Fälle von mehr oder minder vielen Betroffenen immer wieder probiert, weil anders kein Ausweg möglich ist. Dabei vergeht weitere Zeit, und die Schlange verlängert sich weiter, weil die Nachkommenden nicht sogleich erfassen, daß die Zufahrt zum Parkplatz blockiert ist. Die Folge läßt nicht auf sich warten: Termingebundene Verabredungen und wiederkehrende Verpflichtungen mit festen Anfangszeiten (Arbeitsbeginn am Morgen, Vorlesungen und Seminare) können wegen der Schlangenbildung am Parkautomaten oft nicht gehalten werden.

$\mathrm{Zu}$ den durch alle diese Faktoren - alleine oder im Wechselbezug - sich kumulierenden Ärgernissen aufgrund von technischen Regelungen beziehungsweise Unvollkommenheiten kommen noch jene hinzu, die eher finanziellen Kalkülen entspringen: Wer aus privaten oder dienstlichen Gründe den Parkplatz mehrmals am Tage nutzen muß, muß erneut bezahlen. Studierende erwerben mit der Immatrikulation pflichtweise ein sogenanntes Semesterticket, das sie berechtigt, öffentliche Verkehrsmittel in einem mittlerweile nennenswerten Einzugsbereich zu benutzen. Da aber die Benutzung öffentlicher Verkehrsmittel für den Weg zur Universität nicht von allen Ausgangspunkten aus mit vertretbarem zeitlichem Aufwand realisiert werden kann (siehe oben), sieht sich ein Teil der Studierenden gezwungen, trotz des erworbenen Semestertickets mit dem Auto zu fahren und in der Folge auch Parkgebühren zu bezahlen. Somit zahlen beide Personengruppen doppelt.

Unsere Befragungsergebnisse zeigen, in welchem Maße die Befragten Erfahrungen mit den technischen Mängeln sammeln mußten: 
Die Bedienung der Geldautomaten fanden $47 \%$ der Befragten umständlich. Besonders häufig wurden die Parkbelege durch die Automaten nicht ausgedruckt, obwohl das Geld korrekt eingeworfen worden war (bei $12 \%$ der Befragten dreimal und häufiger). ${ }^{22} 47 \%$ haben drei- oder mehrmals erlebt, daß ,ihr“ Geldautomat ganz ausgefallen war. $82 \%$ haben drei- oder mehrmals erlebt, daß die Münzen nicht angenommen wurden. $55 \%$ haben bereits mehrfach an einem Tag Gebühren bezahlen müssen, weil sie den Parkplatz zwischenzeitlich verlassen mußten. Jede(r) Zweite gab an, sich über den krummen Betrag zu ärgern.

Die Quittung für derlei Ungemach erhält in erster Linie die Parkhausfirma, da sie für die Durchführung der Parkplatzbewirtschaftung zuständig ist. Die Urteile fallen um so negativer aus, je mehr der diversen Defekte eine Person erlebt hat und je häufiger dies jeweils der Fall war: ${ }^{23}$

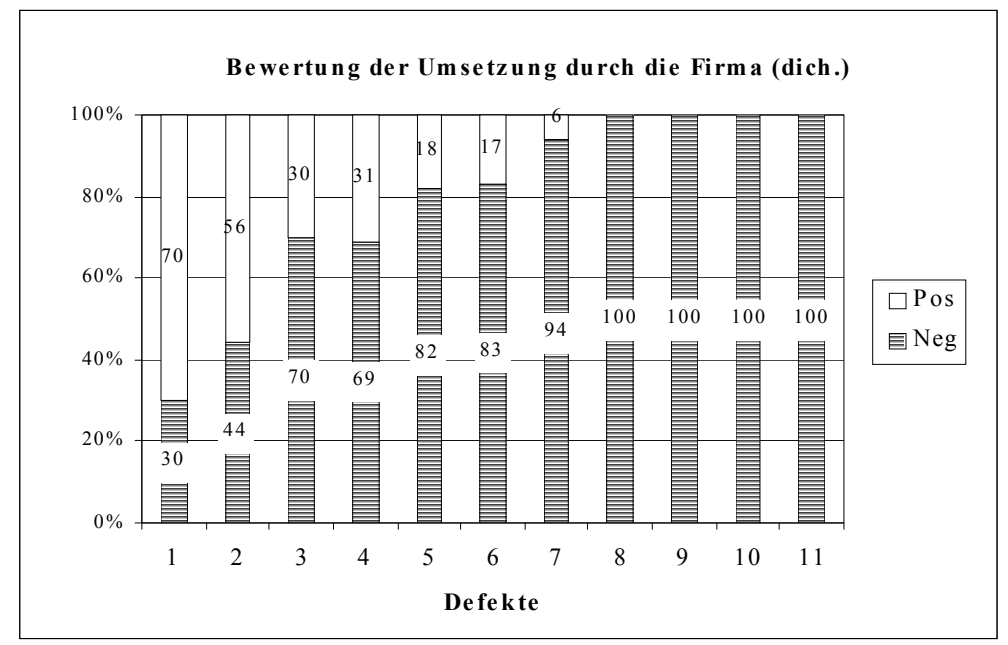

Abbildung 1

In der folgenden Tabelle ist die Vielzahl der Ärgerquellen in nur noch zwei Gruppen zusammengefaßt: ,viele“ und ,wenige“ (Tabelle 1).

\begin{tabular}{|l|l|l|}
\hline $\begin{array}{c}\text { Bewertung der } \\
\text { Parkhausfirma (dichotom) }\end{array}$ & \multicolumn{2}{|c|}{$\begin{array}{c}\text { Zahl der Ärgererfahrungen } \\
\text { (dichotom) }\end{array}$} \\
\hline & wenige (\%) & viele (\%) \\
\hline Ablehnung & 50,0 & 79,8 \\
\hline Zustimmung & 50,0 & 20,2 \\
\hline
\end{tabular}

Tabelle 1

Nemand wird erwarten, daß technische Installationen ohne jede Storung funktionieren. Davon ist auch bei den NutzerInnen auszugehen. Wir haben daher zwei Ärgerfälle pro Item als normal und daher nicht erwähnenswert zugrunde gelegt. Daher werden im folgenden nur solche Ärgerfälle gezählt, die die NutzerInnen dreimal oder häufiger erlebt haben. Das ist sicherlich eher großzügig gerechnet, denn auch dann, wenn Pannen bei einzelnen Items zum ersten Mal auftreten, können sie doch unter entsprechenden Randbedingungen (wie etwa einem hohen und - im Falle von Verspätungen - heiklen Termindruck) zu erheblichem Unmut führen. Das ist um so mehr der Fall, wenn sich vorangegangene Ärgererfahrungen bereits zu einem festen Block von Unmutsgefühlen verdichtet haben.

23 Es wurden zunächst die Antworten auf elf verschiedene Fragen zu praktischen Ärgernissen in 11 Summenscores zusammengefaßt (Abbildung 1). Diese wurden dann ihrerseits zusammengefaßt und mittels des Medians in die Gruppen ,wenige“ und ,viele“ Ärgernisse dichotomisiert (Tabelle 1). 
Hinter diesen Zahlen läßt sich unschwer ein Bewertungsmuster erkennen, das sich so zusammenfassen läßt: ,Wenn schon Parkplatzgebühren, dann muß das System auch funktionieren". Eben dieses ist jedoch in vielerlei Hinsicht und anhaltend nicht der Fall. Die Parkenden fühlen sich dadurch schlecht behandelt. Die Betreiberseite konzentriert sich aus ihrer Sicht darauf, Gebühren zu kassieren, ohne eine angemessene Gegenleistung zu erbringen. Mit anderen Worten: Der Kern der Verärgerung besteht darin, daß die Reziprozitätserwartungen verletzt werden, auf denen die - begrenzte - Bereitschaft der Parkenden aufbaut, sich auf das ungeliebte Reglement einzulassen. Solche Verärgerung fordert ihrerseits zu Vermutungen über die Gründe der Mängel heraus. Die Befragten bieten hier ein ganzes Spektrum an. Es reicht von der sachlichen Kritik, die Parkplatzbewirtschaftung sei „unprofessionell“ durchgeführt worden, über den Vorwurf, mit den gegebenen Maßnahmen würden die Parkenden „,schikaniert“ (,,Die Gebühr ist o.k. Die Groschenbeträge sind eine Schikane, der Automat müßte wechseln") bis hin zu der Mutmaßung, die Regelung diente dazu, über die offiziell geforderten Beträge hinaus ,abzuzocken“ (,Ich empfinde es als halsabschneiderisch, darauf zu spekulieren, daß man DM 1,10 nicht passend hat und so DM 5,- oder ähnlich einschmeißen muß "). ${ }^{24}$

\subsection{Die argumentative Nachvollziehbarkeit des Projekts}

Die Bereitschaft der Befragten, sich auf das ungeliebte Reglement einzulassen, hängt allerdings nicht allein von der Qualität der technischen Regelungen ab. Die betreffenden Mängel und der damit verbundene Ärger bilden zwar gleichsam die Verfügungsmasse der Meinungsbildung im Prozeß des alltäglichen Erlebens. Sie scheinen allerdings in einen weiteren Problemrahmen eingebettet zu sein. Das Problem besteht hier in der Unzulänglichkeit der Begründungen, mit denen die Parkplatzbewirtschaftung als solche gefordert und gerechtfertigt wurde und wird.

Gewiß ist es dabei letztlich nicht entscheidend, was tatsächlich gesagt wurde, sondern welche der Begründungen, die gegeben wurden, tatsächlich durchgedrungen sind und aufgegriffen wurden, und das hat zweifellos auch viel mit der Rezeptionsbereitschaft und den inhaltlichen Voreinstellungen der Befragten selbst zu tun. ${ }^{25}$ Hier kann angenommen werden, daß die zeitgenössischen gesellschaftlichen Rahmenbedingungen (steigende Abgaben für Müll, Wasser; steigende Preise für öffentliche Verkehrsmittel, Benzin und weitere Artikel des täglichen Verbrauchs; Solidaritätsbeitrag; Mißtrauen gegen Politiker wegen Diätenerhöhungen, Bestechlichkeit) bereits eine allgemeine Gereiztheit geschaffen hatten, die nun hier zur Entladung kam. Die erlebten Ärgernisse dürften insoweit auch eine Katalysatorfunktion gehabt haben. Selbst wenn das nicht der Fall gewesen wäre, hätte jedoch alles dafür gesprochen, daß die Befragten erhebliche Zweifel am positiven Sinn der Parkplatzbewirtschaftung gehabt hätten.

Tatsächlich waren ja in Abhängigkeit davon, welcher der beteiligten Akteure sich zur Sache geäußert hatte, ganz unterschiedliche Begründungen bemüht worden, vor allem verkehrspolitische, ökologische und unterschiedliche ökonomische Begründungen, zusätzlich auch stadtentwicklungspolitische und gerechtigkeitspolitische Begründungen. Von

24 Diese Äußerungen sind den zahlreichen Bemerkungen entnommen, die die Befragten auf die offen gestellte Frage niedergeschrieben haben: „Für mich ist die Parkplatzgebühr:......".

25 Zur Problematik der sogenannten Umweltkommunikation siehe etwa de Haan 1995 und Brand/Ederl/Poferl 1997 
einzelnen Akteuren (vor allem der Universität) wurde sogar fast das gesamte Spektrum angeführt. Zudem sind die jeweiligen Begründungen von keinem der Akteure in einer durchstrukturierten und - auf der Basis ausreichender Informationen - auf sorgsame Überzeugung hin angelegten Weise vorgestellt worden. Noch weniger wurden die unterschiedlichen Begründungen der diversen Akteure untereinander abgestimmt oder gar aufeinander bezogen. Und schließlich bestand auf keiner Seite ein ausgearbeitetes Konzept, geschweige denn ein gemeinsames Konzept der beteiligten Akteure zur Schaffung von Akzeptanz, und zwar möglichst im Rahmen eines öffentlichen Verständigungsprozesses zwischen Betreiber- und Nutzerseite. Anstelle dessen wurde das Projekt weithin unter Vermeidung von Öffentlichkeit entwickelt und durchgesetzt. ${ }^{26}$ Dem entsprechen die Ergebnisse der Befragung zu diesem Problembereich. Man könnte die Situation unter dem Begriff des Begründungsnotstands zusammenfassen.

Wir haben zunächst mit drei Fragen danach gefragt, welche Ziele beziehungsweise Motive den Betreibern der Maßnahme zugeschrieben werden. Neben ökonomischen Gründen ${ }^{27}$ und verkehrspolitischen Gründen ${ }^{28}$ haben wir noch „Gerechtigkeitsgründe“ zur Bewertung unterbreitet..$^{29} \mathrm{Daß}$ die Gebühr aus Gerechtigkeitsgründen im Sinne des Verursacherprinzips eingeführt worden sei, nahm nicht einmal ein Viertel aller Befragten an; verkehrspolitische Gründe sahen nur $44 \%$, und selbst die einfachste und vergleichsweise naheliegendste Begründung, eine Einführung aus rein ökonomischen Gründen, unterstellten 1999 lediglich $52 \%{ }^{30}$ (1997 nahmen das immerhin noch 66\% an): (Tabelle 2).

\begin{tabular}{|l|l|l|}
\hline \multicolumn{1}{|c|}{ Die Gebühr wurde eingeführt aus } & Stimme zu & Stimme nicht zu \\
\hline Gerechtigkeitsgründen & $22 \%$ & $78 \%$ \\
\hline verkehrspolitischen Gründen & $44 \%$ & $56 \%$ \\
\hline ökonomischen Gründe & $52 \%$ & $48 \%$ \\
\hline
\end{tabular}

Tabelle 2

Angesichts einer derart breiten Unsicherheit über die Ziele der Betreiber verwundert es nicht, daß auch die Befragten selbst keine überzeugenden Rechtfertigungen sahen: Mehr als drei Viertel der Befragten hielten die Maßnahme aus eigener Sicht weder in ökologischer noch wenigstens in wirtschaftlicher Hinsicht für gerechtfertigt: (Tabelle 3).

Die vergleichsweise meisten publizistischen Aktivitäten entwickelte die Universitätsverwaltung. Auch diese Aktivitäten verblieben jedoch in einem bescheidenen Rahmen. Die Parkplatzbewirtschaftung war hier - allerdings längere Zeit vor ihrer tatsächlichen Einführung - einmal Teilthema einer (stets nur von einem kleinen Teil der Beschäftigten besuchten) Personalversammlung. Ferner waren in einigem zeitlichen Abstand zwei Artikel in der damals zwei- bis dreimonatlich erscheinenden Hauszeitung der Universität („Uni-Schlüssel“) erschienen. Gleichsam als Begleitmaßnahme zur eigentlichen Einführung gab die Universitätsverwaltung schließlich ein Flugblatt heraus.

„Die Gebühr wird alleine aus ökonomischen Gründen erhoben“.

„Die Gebühr wird auch erhoben, um verkehrspolitische Anreize zu schaffen, weniger Auto zu fahren“.

„Die Gebühr wird auch aus Gerechtigkeitsgründen erhoben: Die Kostenverursacher sollen auch die Kosten tragen“.

Die Zustimmung zu den "ökonomischen Gründen"war aber zumindest am eindeutigsten: Die vorgestellten Werte sind aus einer Viererskala dichotomisiert. Bei der Zustimmung konnte aus 2 Varianten gewählt werden: „Stimme voll zu“ und „Stimme eher zu“. Volle Zustimmung erhielten das „Verursacherprinzip“ nur von 4\%, die ,verkehrspolitischen Gründe“ von 6\%, die „,̈konomischen Gründe hingegen von $27 \%$ der Befragten. 


\begin{tabular}{|l|l|l|}
\hline \multicolumn{1}{|c|}{ Für mich ist die Gebühr } & Stimme voll zu & Stimme nicht zu \\
\hline ökologisch gerechtfertigt & $22 \%$ & $78 \%$ \\
\hline wirtschaftlich gerechtfertigt & $24 \%$ & $76 \%$ \\
\hline
\end{tabular}

Tabelle 3

Zusammenfassend läßt sich also festhalten, daß insgesamt eine deutliche Mehrheit der Personen, die auf den Universitätsparkplätzen parken, nicht nachvollziehen kann, warum überhaupt Gebühren erhoben werden.

Es überrascht nicht, daß zwischen diesem offenkundigen Begründungs- und Rechtfertigungsnotstand und der Bewertung der Maßnahme als solcher ein enger Zusammenhang besteht (Abb. 2). Diejenigen mit den geringeren Begründungsproblemen ${ }^{31}$ lehnen die Maßnahme zu $43 \%$ ab, diejenigen mit den größeren Begründungsproblemen jedoch zu 96 \%. Die Zahl der Ärgererfahrungen schlägt demgegenüber weniger stark differenzierend auf die Bewertung der Bewirtschaftung im ganzen durch (74 \% Ablehnung bei „wenigen“ / $81 \%$ bei ,vielen“ Ärgererfahrungen).

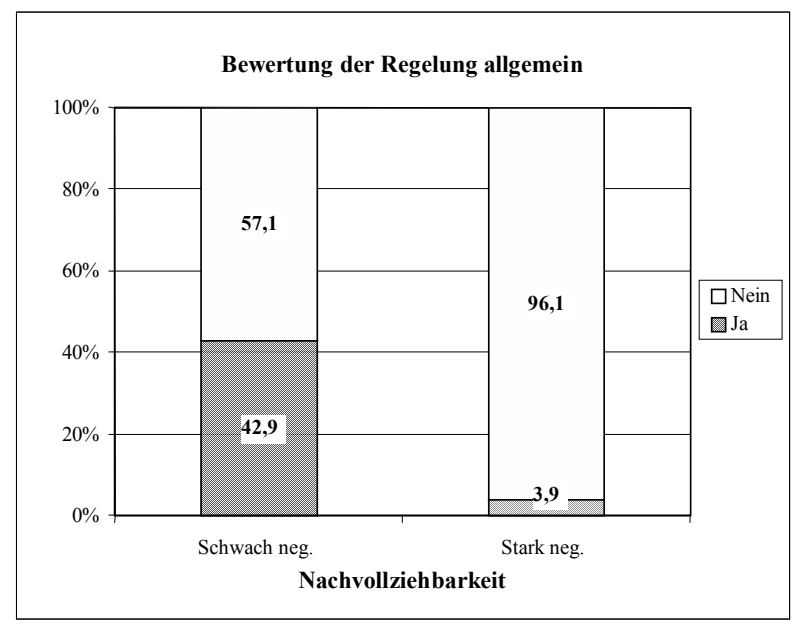

Abbildung 2

Mithin läßt sich sagen, daß die Begründungsproblematik in bezug auf die Bewertung der Bewirtschaftung im ganzen einen relativ eigenständigen Zusammenhang darstellt. Die Betonung liegt dabei allerdings auf „relativ“: Der dargestellte Zusammenhang bildet eine Art allgemeinen Rahmen, innerhalb dessen die täglichen Ärgernisse mit den technischen Mängeln der Parkplatzinstallationen erfahren, erlitten und natürlich auch interpretiert und kritisiert werden.

Beides zusammen verdichtet sich auf seiten der Parkenden zu einem allgemeinen Gefühl, mit der Parkplatzbewirtschaftung einer ungerechtfertigten Zumutung ausgesetzt zu sein. Dem entspricht ein verbreitetes Gefühl der Ohnmacht. Ein erheblicher Teil der Befragten

31 Wir haben hier die Antworten auf sieben verschiedene Fragen zur Nachvollziehbarkeit in Summenscores zusammengefaßt und wiederum den Median als Trennlinie zwischen geringereren Begründungsproblemen (,Schwach negativ“) und größeren Begründungsproblemen (,Stark negativ“) gewählt. 
führt darüber Klage, daß die Besonderheiten ihrer Situation unzureichend beachtet und respektiert würden. $69 \%$ stimmten dem Statement zu „Ich fühle mich bei der Umsetzung der Parkplatzgebühren übergangen“. Die durchaus zugespitzte Formulierung „Für mich ist die Parkplatzgebühr eine ärgerliche Schikane“ ist wohl als Konsequenz solcher Empfindungen zu verstehen. $65 \%$ der Befragten stimmten dem zu.

\section{5 Ökologische Begründungen als Delegitimierungspotentiale}

Eine besondere Rolle spielen dabei die ökologischen Argumente im Spektrum der Begründungen, mit denen die Einführung der Bewirtschaftung begleitet beziehungsweise gerechtfertigt wurde. Anstatt deren Akzeptanz zu befördern, scheinen die ökologischen Argumente zumindest für einen Teil der Befragten genau das Gegenteil zu bewirken. Ökologische Argumente werden von ihnen als Trick verstanden, mit dessen Hilfe die Beschäftigten und Studierenden dazu gebracht werden sollen, einer Sache zuzustimmen, die unzureichend ist. Einigen gilt sie sogar als bloßes Mittel, um ihnen Geld aus der Tasche $\mathrm{zu}$ ziehen, das obendrein für andere Zwecke gebraucht beziehungsweise genutzt wird. ${ }^{32}$ Angesichts der alltäglichen praktischen Mängel wird die Maßnahme zudem noch als unprofessionell umgesetzt und deshalb als besonders ärgerlich empfunden. Der Rekurs auf ökologische Argumente scheint hier weniger deshalb zu Verbitterung zu führen, weil solche Argumente aufgrund fehlenden Umweltbewußtseins für die betreffenden Personen keine sachliche Relevanz und keine persönliche Verbindlichkeit besäßen, sondern weil vermutet wird, daß mit dem Argument „Umwelt“ auf tatsächlich vorhandenen guten Willen und Bürgersinn zurückgegriffen wird, um diese Potentiale zweckentfremdend für andere Ziele einzuspannen. ${ }^{33}$ Ist ein solcher Eindruck erst einmal entstanden, muß jeder Versuch, die Akzeptanz der Bewirtschaftung durch neuerliche Appelle an das Umweltbewußtsein der Betroffenen stärken zu wollen, zum strikten Gegenteil führen: zu weiterer Empörung und in der Folge auch zu weiterer Delegitimierung der Betreiber.

Die gleiche Gefahr besteht auch in einer zweiten Hinsicht. Wie die in den beiden letzten Fußnoten zitierten Äußerungen zeigen, führt der angestaute Ärger auch zu ungerechter Kritik, zum Teil auch zu unsachlicher Kritik. Das gilt nicht nur für die Problematik der Begründungen der Parkplatzbewirtschaftung, sondern auch für die Kritik an den technischen Installationen.

Eine ganze Reihe von Kritiken der NutzerInnen sind in diesem Sinne von der Parkhausgesellschaft öffentlich relativiert oder auch zurückgewiesen worden (Uni-Schlüssel $\mathrm{H}$. 45/1997). Der Kern der Zurückweisungen lautete, es handele sich weithin nicht um Schwächen der Technik, sondern um Bedienungsfehler oder überzogene Erwartungen, mithin um Schwächen der NutzerInnen. Tatsächlich wird sich ein Teil der beschriebenen

\footnotetext{
32 Auf die 1997 gestellte Frage „Wenn Sie sich geärgert haben: Was hat Sie am meisten gestört“? notierten Befragte zum Beispiel: „Die Argumentationsweise. Die Gebühren werden nicht aus ökologischen Gründen erhoben...Sowohl im ,Uni-Schlüssel 'als auch im Flugblatt wird das Geld als 1. Grund genannt. Kein Betrieb lastet seinen Mitarbeitern die Betriebskosten an! Etwas überzogen: Müssen wir nächstens 50 Pfennig in die Toilettentür stecken, um die Kosten dieser Einrichtung der Forschung zugängig zu machen? “. Eine andere Person schrieb: ,,Unter dem Deckmäntelchen der Ökologie und der so teuren Parkplätze versucht die Uni uns diese Geschichte schmackhaft zu machen, wobei doch jeder weiß, daß a) die Uni Geld braucht - für alles mögliche, nicht jedoch nur, um die Parkplätze zu unterhalten; ist ja auch legitim, bloß warum dann das Herumgelüge - und b) daß das der Uni zur Verfügung stehende Geld teilweise unsinnig rausgeschmissen wurde... “.

33 Etwa im Sinne einer weiteren Antwort auf die in der vorangehenden Fußnote vorgestellte Frage: „Ökologisches Gelaber. Parkgebühren haben doch nix mit Umweltbewußtsein zu tun".
} 
Mißlichkeiten und Ärgernisse bei entsprechend informiertem, vorausplanendem und geschicktem Verhalten reduzieren lassen. Da dies jedoch keineswegs für alle auftretenden Probleme gilt und da auch diese Probleme bis heute fortdauern und vergleichsweise gewichtiger sind (Schranke öffnet nicht, kein Ausdruck, Münzen fallen durch), erscheint die Kritik der Betreibergesellschaft an den Parkenden aus deren Sicht leicht als Versuch, von der eigenen Verantwortung für die offenkundigen Schwächen abzulenken und den NutzerInnen den Schwarzen Peter zuzuschieben, das heißt jenen, die ohnehin die Opfer sind. Es handelt sich darin um einen klassischen Fall von „blaming the victim“ (Ryan 1971). Auch ein solches Vorgehen ist also gänzlich ungeeignet, die Akzeptanz der Sache zu erhöhen. Es heizt anstelle dessen den Ärger eher noch an und führt darüber zu weiterer Delegitimierung der Betreiber.

Im übrigen ist auch in der Gruppe jener, die vergleichsweise wenige Ärgererfahrungen mit der Technik gesammelt haben, und die insofern einigermaßen zurecht kommen, die Ablehnung der Maßnahme im ganzen mit 74 \% schon sehr hoch. Selbst wenn sich also alle, die sich jetzt noch mit der Technik schwertun, persönlich besser darauf einstellen wollten und könnten, würden sich die Akzeptanzprobleme keineswegs erübrigen. Die Vorbehalte gegen die Maßnahme haben eben schon ein hohes Niveau, bevor der Alltag der Parkplatznutzung überhaupt beginnt. Die unzulänglichen Begründungen der Notwendigkeit und Sinnhaftigkeit der ganzen Maßnahme ist gewiß nicht der einzige, gewiß aber ein wesentlicher Erklärungsgrund dieses Sachverhalts.

Eine nennenswerte Überwindung der Akzeptanzdefizite müßte den Tatbestand zum Ausgangspunkt nehmen, daß der bisherige Verlauf der Dinge in doppelter Weise die mit den Angemessenheitsvorstellungen der Parkenden verbundenen Reziprozitätserwartungen enttäuscht hat. Hier bedarf es folglich eines doppelten Ausgleichs, der in der gegebenen Konstellation nur dann zum Erfolg führen kann, wenn ihn die Betreiber primär als ihre Bringschuld ansehen: durch Überwindung der wichtigsten Ärgernisse bei der alltäglichen Parkplatznutzung und durch die Präsentation eindeutiger und überzeugender Begründungen für die Notwendigkeit der Bewirtschaftung als ganzer und die Sinnhaftigkeit ihres konkreten Zuschnitts.

Gelingt das nicht, so spricht einiges dafür, daß nicht nur der vorhandene Ärger bestehen bleibt, sondern daß der konkrete Fall darüber hinausgehende Fernwirkungen in dem Sinne entwickelt, daß ökologische Argumente auch in anderen Fragen staatlich oder in sonstiger Weise zugemuteter Veränderungen bestehender Routinen bei den davon betroffenen Universitätsangehörigen nicht etwa mehr Aufgeschlossenheit nach sich ziehen, sondern vermehrtes Mißtrauen. Das dürfte um so mehr der Fall sein, wie die Betroffenen dabei auch noch zur Kasse gebeten werden.

Der vielfach notierte und beklagte Rückgang umweltbezogener Handlungsbereitschaften von Bürgerinnen und Bürgern dürfte sich nicht zuletzt aus der Kumulation von Erfahrungen des hier skizzierten Typs in verschiedenen Bereichen ihres eigenen Alltags und in der Beobachtung des Alltags der Politik erklären. Auch in diesem größeren Rahmen würde daher gelten, daß eine Besserung nur denkbar ist, wenn bereits verletzte Angemessenheitsvorstellungen breiter zur Kenntnis genommen, stärker respektiert und schließlich durch geeignete praktische Maßnahmen ausgeglichen würden, oder anders ausgedrückt: wenn Ökologie und Gerechtigkeit nicht als zwei verschiedene Themen, sondern als zwei 
Seiten einer Medaille verstanden und gehandhabt würden - vor allem in der politischen Praxis, aber auch in der umweltsoziologischen und -psychologischen Forschung. 


\section{Literaturverzeichnis}

Bierhoff, H.W. (1992): Prozedurale Gerechtigkeit. Das Wie und Warum der Fairness. In: Zeitschrift für Sozialpsychologie, H. 23, S. 163-178

Brand, K.-W. (1997): Probleme und Potentiale einer Neubestimmung des Projekts der Moderne unter dem Leitbild ,Nachhaltige Entwicklung'. In: Ders. (Hrsg.): Nachhaltige Entwicklung. Eine Herausforderung an die Soziologie. S. 9-34. Opladen: Leske + Budrich,

Brand, K.-W.; Eder, K.; Poferl, A. (1997): Ökologische Kommunikation in Deutschland. Opladen: Westdeutscher Verlag

de Haan, G. (Hrsg.) (1995): Umweltbewußtsein und Massenmedien. Perspektiven ökologischer Kommunikation. Berlin: Akademie Verlag

de Haan, G.; Kuckartz, U. (1996): Umweltbewußtsein. Denken und Handeln in Umweltkrisen. Opladen: Westdeutscher Verlag

Fuhrer, U.; Wölfing, S. (1997): Von den sozialen Grundlagen des Umweltbewußtseins zum verantwortlichen Umwelthandeln. Die sozialpsychologische Dimension globaler Umweltproblematik. Bern u.a.: Huber

Fuhrer, U. (1995): Ökologisches Handeln als sozialer Prozeß. Basel: Birkhäuser

Gorr, H (1996): Die Logik der individuellen Verkehrsmittelwahl. Theorie und Realität des Entscheidungsverhaltens im Personenverkehr. Gießen: Focus

Homburg, A.; Matthies (1998): Umweltpsychologie. Umweltkrise, Gesellschaft und Individuum. Weinheim und Bonn: Juventa

(HUI) Bundeskoordination Studentischer Ökologiearbeit (Hrsg.) (1996): HochschulUmwelt-Info, Nr. 6, S. 30. München

Jurczyk, K.; Rerrich, M. (Hrsg.) (1993): Die Arbeit des Alltags. Beiträge zu einer Soziologie der alltäglichen Lebensführung. Freiburg: Lambertus

Poferl, A.; Schilling, K.; Brand, K.-W. (1997): Umweltbewußtsein und Alltagshandeln. Eine empirische Untersuchung sozial-kultureller Orientierungen, hrsg. v. Umweltbundesamt. Opladen: Leske und Budrich

Preisendörfer, P.; Franzen, A. (1996): Der schöne Schein des Umweltbewußtseins. Zu den Ursachen und Konsequenzen von Umwelteinstellungen in der Bevölkerung. In: Diekmann; A., Jaeger, C.: Umweltsoziologie. Sonderheft 36 der Kölner Zeitschrift für Soziologie und Sozialpsychologie, S. 219-244

Preisendörfer, P. (1996): Umweltbewußtsein in Deutschland. Bonn: Bundesministerium für Umwelt, Naturschutz und Reaktorsicherheit

Reichle, B. (Hrsg.) (1998): Verantwortung, Gerechtigkeit und Moral. Zum psychologischen Verständnis ethischer Aspekte des menschlichen Verhaltens. Weinheim: Juventa

Schahn, J.; Giesinger, T. (Hrsg.) (1993): Psychologie für den Umweltschutz, Weinheim: Psychologie Verlags Union

Warsewa, G. (1997): Moderne Lebensweise und ökologische Korrektheit. Zum Zusammenhang von sozialem und ökologischem Wandel. In: Brand, K.-W. (Hrsg.): Nachhaltige Entwicklung. Eine Herausforderung an die Soziologie. S. 195-210. Opladen: Leske + Budrich 



\section{a $r t e c$}

\section{Ziele und Umfeld}

artec ist eine grundlagenorientierte Forschungseinrichtung. Unser Thema sind Gestaltungsprobleme im Verhältnis von Arbeit, Technik und Umwelt.

artec existiert seit 1989 als Zentrale Wissenschaftliche Einrichtung (ZWE) der Universität Bremen.

artec ist multidisziplinär zusammengesetzt. Seine Angehörigen kommen zur Hälfte aus Ingenieurwissenschaften und Informatik und zur Hälfte aus verschiedenen Sozialwissenschaften.

artec-Forschungsergebnisse aus beiden Bereichen fließen regelmäßig in die Lehre ein.

artec ist besonders an praxisnahen Formen der Gewinnung von Forschungsergebnissen und an deren Rückvermittlung in die Praxis interessiert.

artec kooperiert in diesem Sinne mit außeruniversitären Einrichtungen und Betrieben.

Im Mittelpunkt der artec-Forschung stehen gegenwärtig Probleme gestiegener Komplexität in der Arbeits- und Technikgestaltung als Folgen verkürzter Entwicklungszeiten und erweiterter Produktverantwortung (vor allem Anforderungen des Umweltschutzes).

Anschrift: Forschungszentrum Arbeit und Technik (artec), EnriqueSchmidt-Str. (SFG), Postfach 3304 40, D-28334 Bremen, Tel. 0421 / 2182435, Fax 0421 / 218-4449, http://www.uni-bremen.de/ artec/ 



\title{
Forschungszentrum Arbeit und Technik (artec)
}

\author{
Enrique-Schmidt-Straße 7, Seminar- und Forschungsverfügungsgebäude (SFG),
} Postfach 3304 40, D-28334 Bremen,

Tel. 0421 / 218-2435, Fax 0421 / 218-4449, http://www.uni-bremen.de/ artec/

\section{artec-papers}

kostenlos unter obiger Adresse zu beziehen, soweit nicht anders vermerkt.

Nr. 1 (vergriffen)

Forschungszentrum Arbeit und Technik (artec)

Entstehung und Programm: Kurzfassung 1990. 16 S.

Nr. 2 (vergriffen)

Lange, Hellmuth / Senghaas-Knobloch, Eva

Projekt: Gestaltungsdebatten und Partizipationsansätze für EDV-gestützte Techniken im industriegesellschaftlichen Vergleich.

1990. $38 \mathrm{~S}$.

\section{Nr. 3}

Friedrich, Jürgen / Lange, Hellmuth /

Nake, Frieder u.a.

Projekt: Kooperative Gestaltung informationstechnischer Arbeitsmittel für kooperatives Arbeiten: Neuartige Zugänge zu Literaturbeständen. 1990. $43 \mathrm{~S}$.

\section{Nr. 4}

Cords, Dagmar / Müller, Wilfried

Projekt: Arbeitsorientierte Technikbewertung. CAD-Systemanpassung durch Konstrukteur/Innen. 1990. $22 \mathrm{~S}$.

\section{Nr. 5 (vergriffen)}

Hellige, Hans Dieter

Projekt: Technikgenese, Entwicklungsmuster und Gestaltungsprinzipien von Daten-, Rechner- und Informationsnetzen im Vergleich mit traditionellen Telekommunikationstechniken.

1990. $24 \mathrm{~S}$.

\section{Nr. 6}

Creutz, Annemarie / Reinhardt, Margarethe / Fierek, Nicole / Volmerg, Birgit (Projektleiterin) Projekt: Geschlechtersozialisation und soziale Herkunft in ihrer Bedeutung für Lernchancen und Lernhindernisse im Informatikunterricht der Gymnasialen Oberstufe. 1990. $20 \mathrm{~S}$.

\section{Nr. 7}

Heimbucher, Achim

Zur Geschichte des Gestaltungsbegriffs in der Kunst. 1990. $26 \mathrm{~S}$.
Nr. 8

\section{Hellige, Hans Dieter}

Leitbilder und historisch-gesellschaftlicher Kontext der frühen wissenschaftlichen Konstruktionsmethodik. 1991. $53 \mathrm{~S}$.

\section{Nr. 9 (vergriffen)}

\section{Senghaas-Knobloch, Eva}

Was heißt hier Gestaltung? Propädeutika für eine deutsch-deutsche Arbeit- und Technikforschung. 1991. $29 \mathrm{~S}$.

(erschienen in: Werner Fricke (Hg.), Jahrbuch Arbeit und Technik des Landes Nordrhein-Westfalen, Bonn 1991, S. 84-94)

Nr. 10 (vergriffen)

Lange, Hellmuth

Arbeits- und Technikgestaltung unter dem Primat der Politik in der ehemaligen DDR. 1991. $22 \mathrm{~S}$.

(erschienen in: Werner Fricke (Hg.), Jahrbuch Arbeit und Technik des Landes Nordrhein-Westfalen, Bonn 1991, S. 95-104)

\section{Nr. 11}

Müller, Wilfried / Cords, Dagmar

Gestaltungsperspektiven und Rationalisierungsverständnis von Ingenieuren.

1991. $14 \mathrm{~S}$.

Nr. 12

\section{Cords, Dagmar}

Perspektiven der Organisationsgestaltung bei CAD stehen noch aus.

1991. $19 \mathrm{~S}$.

\section{Nr. 13}

\section{Hellige, Hans Dieter}

Bibliographie der Nachschlagewerke für die Gebiete Elektronik, Kommunikationstechnik, Informationstechnik und Produktionstechnik. 1991. $39 \mathrm{~S}$.

Nr. 14

Cords, Dagmar / Müller, Wilfried

Betriebliche CAD-Systemanpassung als Aufgabe für Konstruktionsmitarbeiter/innen. 1991. $14 \mathrm{~S}$. 
Nr. 14 E

\section{Cords, Dagmar / Müller, Wilfried}

In-company adaption of CAD system by end users. (Englische Fassung von Nr. 14).

1991. $15 \mathrm{~S}$.

\section{Nr. 15}

\section{Hellige, Hans Dieter}

Militärische Einflüsse auf Leitbilder, Lösungsmuster und Entwicklungsrichtungen der Computer-

kommunikation.

1992. $42 \mathrm{~S}$.

\section{Nr. 16 (vergriffen)}

Senghaas-Knobloch, Eva

Arbeit und die Aufgabe zukunftsfähiger gesellschaftlicher Entwicklung in der neuen Bundesrepublik.

1992. $77 \mathrm{~S}$.

(erschienen in: Hansgert Peisert, Wolfgang Zapf (Hg.), Gesellschaft, Demokratie und Lebenschancen. Festschrift für Ralf Dahrendorf, Stuttgart 1994, S. 313-330)

\section{Nr. 17}

Müller, Dieter / Bruns, Friedrich Wilhelm

Hypermedia als Simulationswerkzeug. 1992. $29 \mathrm{~S}$.

\section{Nr. 18}

\section{Lange, Hellmuth}

DDR-Intelligenz auf dem Weg ins berufliche Abseits? Beeinträchtigung beruflicher Identität in der DDR als Hemmschuh im Transformationsprozeß. 1992. $35 \mathrm{~S}$.

\section{Nr. 19}

\section{Bruns, Friedrich Wilhelm}

Über die Rückgewinnung von Sinnlichkeit:

Eine neue Form des Umgangs mit Rechnern. 1993. $13 \mathrm{~S}$.

\section{Nr. 20}

\section{Hellige, Hans Dieter}

Akteure, Leitbilder und Entwicklungslinien in der Geschichte der Computerkommunikation. 1993. $25 \mathrm{~S}$.

\section{Nr. 20 E}

\section{Hellige, Hans Dieter}

Actors, Visions and Developments in the History of Computer Communications.

(Englische Fassung von Nr. 20).

1993. $25 \mathrm{~S}$.

\section{Nr. 21}

Bruns, Friedrich Wilhelm / Heimbucher, Achim / Müller, Dieter

Von der Desktopperspektive zur Werkstattperspektive. Ansätze zu einer erfahrungsorientierten Gestaltung von Rechnersystemen für die Produktion. 1993. $66 \mathrm{~S}$.

\section{Nr. 22}

\section{Müller, Dieter}

Application Frameworks für die Entwicklung interaktiver Anwendungen mit multisensorischen Benutzungsschnittstellen. Ansätze einer prototypischen Realisierung von Rechnersystemen für die Produktion. 1993. $29 \mathrm{~S}$.

\section{Nr. 23}

Hoffmann, Fernand / Spaus, Jean-Marie

Werkstofforientiertes Programmieren: Entstehung, Ansätze, Erfolge und Probleme. 1993. $40 \mathrm{~S}$.

\section{Nr. 24}

\section{Hellige, Hans Dieter}

Von der programmatischen zur empirischen Technikgeneseforschung. Ein technik-historisches Analyseinstrumentarium für die prospektive Technikbewertung. 1993. $59 \mathrm{~S}$.

\section{Nr. 25}

\section{Lange, Hellmuth}

Wissenschaftlich-technische Fachkräfte im Wettlauf mit der Zeit. Probleme des beruflichen Übergangs vom DDR-Sozialismus zur Marktwirtschaft der BRD. 1993. $42 \mathrm{~S}$.

\section{Nr. 26}

Nake, Frieder / Schelhowe, Heidi

Vom instrumentellen Medium. Kooperation in der Software-Entwicklung unter konfligierenden Leitbildern. 1993. $127 \mathrm{~S}$.

\section{Nr. 27}

Engeln, Olaf von / Fischer, Dirk / Grislawski, Ralf u.a.

Simulation in Produktion und Logistik. Ein studentisches Projekt.

1993. $31 \mathrm{~S}$. 
Nr. 28

Hildebrandt, Eckart / Müller, Wilfried (Hg.)

Zur umweltverträglichen Gestaltung technischer

Projekte.

1993. $129 \mathrm{~S}$.

Nr. 29

Senghaas-Knobloch, Eva / Nagler, Brigitte

Konflikte und moralische Dilemmata in der betriebli-

chen Lebenswelt. Entwurf zu einer empirischen Studie. 1993. $27 \mathrm{~S}$.

Nr. 30

\section{Gust, Rolf}

'Kooperation' und 'Erfahrung' in der hochautomatisierten Produktion der stoffumwandelnden Industrie - Ein Problemaufriß.

1993. $29 \mathrm{~S}$.

Nr. 31

\section{Cords, Dagmar}

Gegenstandsbereiche der betrieblichen CAD-

Systemanpassung: Ein Strukturierungsvorschlag aus

Anwendersicht.

2. erw. Auflage. 1994. 14 S.

Nr. 32

Frech, Ulrich / Müller, Wilfried

Teamarbeit ist kein Software-Problem. Vorläufige

Ergebnisse einer explorativen Studie zur Gewinnung von Gestaltungsalternativen.

1994. $19 \mathrm{~S}$.

Nr. 33

Hellige, Hans Dieter (Hg.)

Leitbilder der Informatik- und Computer-Entwicklung. Eine Tagung der Fachgruppe Historische Aspekte von Informatik und Gesellschaft der Gesellschaft für Informatik und des Deutschen Museums. München 4.6. Oktober 1993, Tagungsband. 1994. 478 S. (nur als Diskette erhältlich)

Nr. 34

\section{Müller, Wilfried}

CAD-Systemanpassung als Gegenstand betrieblicher Aushandlungsprozesse. Ergebnisse einer empirischen Studie in kleineren und mittleren Betrieben des Maschinenbaus.

1994. $84 \mathrm{~S}$.

Nr. 35 (vergriffen)

Dohms, Annette / Nagler, Brigitte /

Senghaas-Knobloch, Eva

Herausforderung Gruppenarbeit - Streiflichter auf

Erlebnisperspektiven. Ein Werkstattbericht.

1995. 19 S.
Nr. 36 (vergriffen)

Senghaas-Knobloch, Eva / Nagler, Brigitte /

Dohms, Annette

Industrielle Gruppenarbeit aus der Erlebnisperspektive. Berufliche Selbstbilder im Wandel.

1995. $25 \mathrm{~S}$.

\section{Nr. 37}

Gust, Rolf

Verbreitungsgrad und Probleme des Einsatzes moderner Qualitätsmanagementmethoden.

Literaturbericht im Rahmen des Modellversuchs „Q2 Qualifikation zur Qualität. 1995. $20 \mathrm{~S}$.

Nr. 38

Modrow-Thiel, Brita

Subjektivität im Arbeitshandeln als neue Perspektive der Personalarbeit.

1995.

Nr. 39

\section{Lange, Hellmuth}

Über das ökologische Potential der automobilen

Lebensweise. Umwelthandeln zwischen Einsicht und alltäglichen Zwängen.

1995. $28 \mathrm{~S}$.

Nr. 40

Hellige, Hans Dieter

Technikgestaltung: Ein Begriff als Programm, seine Geschichte, Systematik und Problematik. 1995. $28 \mathrm{~S}$.

Nr. 41 (vergriffen)

\section{Gust, Rolf / Müller, Wilfried}

Methoden der Qualitätssicherung in kleinen und mittleren Betrieben des Maschinen- und Anlagenbaus. 1996. 34 S.

Nr. 42

Brauer, Volker

Gestenerkennung mit einem Datenhandschuh. 1996. $13 \mathrm{~S}$.

Nr. 43

Bruns, Friedrich Wilhelm / Müller, Dieter

Lernförderliche Übergänge zwischen gegenständlichen und abstrakten Modellen technischer Systeme. 1996. $21 \mathrm{~S}$.

Nr. 44

Bruns, Friedrich Wilhelm

Grasping, Communicating, Understanding. 1996. $18 \mathrm{~S}$. 
Nr. 45 (vergriffen)

\section{Gust, Rolf}

Computerunterstütztes Lernen am Lernort 'Arbeitsplatz' in kleinen und mittleren Unternehmen: Voraussetzungen, Bedingungen und Probleme.

1996. $20 \mathrm{~S}$.

\section{Nr. 46}

Bruns, Friedrich Wilhelm / Volker Brauer

Bridging the Gap between Real and Virtual Modelling. A new Approach to Human- Computer Interaction. 1996. $9 \mathrm{~S}$.

\section{Nr. 47}

\section{Frech, Ulrich / Müller, Wilfried}

Die Grenzen der „Irgendwie“ - Kommunikation. Vernetzte CAD-Systeme und Kooperation in Kleinund Mittelunternehmen.

1996. $79 \mathrm{~S}$.

\section{Nr. 48}

\section{Hellige, Hans Dieter}

Bibliographie der Nachschlagewerke für die Gebiete Elektronik, Kommunikationstechnik, Informationstechnik und Produktionstechnik, 2. Auflage. 1996. $51 \mathrm{~S}$.

\section{Nr. 49}

\section{Peters, Jörg}

Technikfolgenabschätzung und politische Verantwortung des Parlaments. Das Büro für Technikfolgenabschätzung beim Deutschen Bundestag in einer politikwissenschaftlichen Wirkungsanalyse. 1996. $89 \mathrm{~S}$.

\section{Nr. 50}

artec-Forschungsprogramm 1995-1998 - Aktualisierte Fassung

Kooperative Bewältigung neuer Komplexität in der Gestaltung von Arbeit und Technik 1997. $116 \mathrm{~S}$.

\section{Nr. 51}

\section{Kumbruck, Christel}

Gestaltung von Technik zur Kooperationsunterstützung.

1997. $25 \mathrm{~S}$.

\section{Nr. 52 (vergriffen)}

\section{Gust, Rolf / Müller, Wilfried}

Arbeitsplatznahe Weiterbildung durch computergestützte Lernsoftware. Erste Ergebnisse am Beispiel neuer Methoden des Qualitätsmanagements. 1997. $19 \mathrm{~S}$
Nr. 53

\section{Perret, Bernard}

Beyond unemployment - systemic social issues of work transformations.

1997. $15 \mathrm{~S}$.

\section{Nr. 54}

Brauer, Volker / Bruns, Friedrich Wilhelm

Hornecker, Eva / Robben, Bernd / Rosch, Hartmut / Rügge, Ingrid / Schäfer, Kai

Vom Bildschirm zum Handrad. Computer(be)nutzung nach der Desktop-Metapher, Workshop, 6.-7. Oktober 1997, Workshop-Abstracts, $35 \mathrm{~S}$.

\section{Nr. 55}

\section{Hellige, Hans Dieter}

Werkstoffwahl, energetische Dimensionierung und andere umweltrelevante Designkriterien in der Problemstruktur der Telefaxtechnik. 1997. $21 \mathrm{~S}$.

Nr. 56

Brauer, Volker / Bruns, Friedrich Wilhelm / Schäfer, Kai

Rechnergestützte Übergänge zwischen gegenständlichen und abstrakten Modellen produktionstechnischer Systeme. Erster und zweiter Zwischenbericht zum DFG-Forschungsprojekt RUGAMS. 1997. $54 \mathrm{~S}$.

\section{Nr. 57}

\section{Zoll, Rainer}

Zivildienst als Legitimation für ein Bürgereinkommen. 1998. $20 \mathrm{~S}$.

\section{Nr. 58 (vergriffen)}

\section{Senghaas-Knobloch, Eva}

Von der Arbeits- zur Tätigkeitsgesellschaft? Politikoptionen und Kriterien zu ihrer Abschätzung. 1998. $32 \mathrm{~S}$.

veröffentlicht in Feministische Studien, Heft 2/1998

\section{Nr. 59}

Bruns, Friedrich Wilhelm / Hornecker, Eva /

Robben, Bernd / Rügge, Indrid

Vom Bildschirm zum Handrad. Computer(be)nutzung nach der Desktop-Metapher, Workshop, 6.-7. Oktober 1997, Workshop-Dokumentation.

1998. $256 \mathrm{~S}$. 
Nr. 60

Becker, Kai M. / Bleich, Olaf / Dogan, Özden /

Duval, Cédrik / Faust, Martin / Jabaly, Dawoud /

Klemm, Michael / Manos, Kanstantinos / Mühlhaus, Sigrid / Nolte, Markus / Piedboef, Keith-André

RUMpv: Rechnergestützte Übergänge zwischen

Modellen physikalischer und virtueller Realität. 1998. 594 S.

Nr. 61

Senghaas-Knobloch, Eva

Die Internationale Arbeitsorganisation vor neuen

Herausforderungen der Globalisierung?

1998. $38 \mathrm{~S}$.

Nr. 62

Hornecker, Eva / Robben, Bernd

Lernen und Begreifen - Pneumatikunterricht in einer

Real Reality-Umgebung.

1998

Nr. 63

Dirks, Jan

„Universales Positives Regieren“ jenseits des Nationalstaates. Zwei Fälle im maritimen Sektor.

1998. $57 \mathrm{~S}$.

Nr. 64

Manske, Fred / Moon, Yonggap

Innovation auf Koreanisch. Elektronische Kommunikation in der koreanischen und deutschen Automobilin-

dustrie.

1998. $261 \mathrm{~S}$.

Nr. 65

Hornecker, Eva / Schäfer, Kai

Gegenständliche Modellierung virtueller Welten. 1998. $10 \mathrm{~S}$.

Nr. 66

Moldaschl, Manfred / Nagler, Brigitte /

Senghaas-Knobloch, Eva

Subjektivität, ökonomische Verwertung und Arbeitskultur. Zwei Beiträge zu den Auswirkungen neuer Managementkonzepte.

1999. $33 \mathrm{~S}$.

Nr. 67

Schäfer, Kai / Bruns, Friedrich Wilhelm /

Volker Brauer

Rechnergestützte Übergänge zwischen gegenständlichen und abstrakten Modellen produktionstechnischer Systeme. Dritter Zwischenbericht zum DFG-

Forschungsprojekt RUGAMS.

1999. $57 \mathrm{~S}$.
Nr. 68

Nagler, Brigitte / Hartmann, Wiebke /

Senghaas-Knobloch, Eva

Cultural change includes a political dimension. 1999. $19 \mathrm{~S}$.

Nr. 69

Dolata, Ulrich

Hot House. Konkurrenz, Kooperation und Netzwerkbildung in der Biotechnologie.

1999.

Nr. 70

artec-Forschungsprogramm 1999-2000

Kooperation als fach- und professionsübergreifende Gestaltungsaufgabe.

1999.

\section{Nr. 71}

Modrow-Thiel, Brita

Telearbeit und Telekooperation als Herausforderung für die arbeitswissenschaftliche Gestaltungsperspektive.

1999. $22 \mathrm{~S}$.

Nr. 72

Lange, Hellmuth / Rau, Andreas

Akzeptanz der Parkplatzbewirtschaftung auf den

Plätzen der Universität im April 1999

1999. $53 \mathrm{~S}$. 